\title{
THE LAST PROBLEM OF HARALD BOHR
}

\author{
JEAN-PIERRE KAHANE
}

(Received 2 September 1987)

Communicated by W. Moran

\begin{abstract}
The first and last papers of Harald Bohr deal with ordinary Dirichlet series $\sum_{1}^{\infty} a_{n} n^{-s}$ and their order (or Lindelöf) function $\mu(\sigma)\left(=\inf \left\{\alpha ; f(\sigma+i t)=o\left(|t|^{\alpha}\right)\right\}\right)$. The Lindelöf hypothesis is $\mu(\sigma)=\inf \left(0, \frac{1}{2}-t\right)$ when $a_{n}=(-1)^{n}$. Are there ordinary Dirichlet series with $-1<\mu^{\prime}(\sigma)<0$ for some $\sigma$ ? A negative answer would imply Lindelöfs hypothesis. This is the last problem of Harald Bohr. This paper gives (1) a review on Bohr's results on ordinary Dirichlet series; (2) a review on results of the author and of Queffelec on "almost sure" and "quasi sure" properties of series $\sum_{1}^{\infty} \pm n^{-s}$ with the solution of a previous problem of Bohr; (3) the following answer to the last problem: $\mu^{\prime}(\sigma)$ can approach $-\frac{1}{2}$, and necessarily $\mu\left(\sigma+\mu(\sigma)+\frac{1}{2}\right)=0$. The characterization of the order functions of ordinary Dirichlet series remains an open question.
\end{abstract}

1980 Mathematics subject classification (Amer. Math. Soc.) (1985 Revision): 30 B 50.

The centenary of Harald Bohr (1887-1951) provides an opportunity to revisit his works. My purpose is to consider his first and last papers [1], [2], [3], [4] which deal with Dirichlet series of the form

$$
\sum_{1}^{\infty} a_{n} n^{-s} \quad(s=\sigma+i t)
$$

("ordinary Dirichlet series" in the terminology of Hardy and Riesz [5]). I shall describe his main results and mention two questions of his, one already solved 14 years ago, the other-the last problem of Harald Bohr-being the main topic of this report. Then I shall indicate how his main results can be derived and the easy question solved by using almost sure and quasi-sure

(C) 1989 Australian Mathematical Society $0263-6115 / 89 \$ A 2.00+0.00$ 
properties of series of the form

$$
\sum_{1}^{\infty} \pm n^{-s}
$$

already introduced in [8] and studied by $H$. Queffelec [11]. Finally, I shall give partial answers to the "last problem." This work was done during my visit to the Centre for Mathematical Analysis at the A.N.U. in Canberra.

\section{Bohr's results and problems}

In his thesis [1], written in 1909, H. Bohr starts from the following known results.

(a) If (1) converges for $s=s_{0}=\sigma_{0}+i t_{0}$, it converges absolutely when $\sigma>\sigma_{0}+1$ and the sum is bounded whenever $\sigma \geq \sigma_{0}+1+\varepsilon(\varepsilon>0)$;

(b) (Jensen) it converges in the half plane $\sigma>\sigma_{0}$ (uniformly in each angle $\left.\left|\arg \left(s-s_{0}\right)\right| \leq \pi / 2-\varepsilon(\varepsilon>0)\right)$ and its sum $f(s)$ satisfies

$$
f(\sigma+i t)=O(|t|) \quad(|t| \rightarrow \infty)
$$

whenever $\sigma>\sigma_{0}$ (uniformly if $\sigma \geq \sigma_{0}=\varepsilon, \varepsilon>0$ given);

(c) (Schnee) whenever $f(s)$ can be continued analytically into a half-plane $\sigma>\sigma_{1}-h(h>0)$ and satisfies

$$
f\left(\sigma_{1}+i t\right)=O\left(|t|^{\varepsilon}\right) \quad(|t| \rightarrow \infty)
$$

for each $\varepsilon>0$, (1) converges for $\sigma>\sigma_{1}$ (this is not an easy result; the best proof is due to $\mathrm{H}$. Helson [7]).

In what respect are the convergence and summability of the series (1) reflected in the properties of $f(s)$ as an analytic function? This is the main theme of the thesis. In particular, what is the relation between the convergence or summability properties of (1) and the "order function" (now called the Lindelöf function) $\mu(\sigma)$ defined as

$$
\mu(\sigma)=\inf \left\{\beta>0 \mid f(\sigma+i t)=O\left(|t|^{\beta}\right)\right\} \quad(|t| \rightarrow \infty)
$$

whenever the function $f$ can be defined in a vertical half-plane containing $\sigma$ by analytic continuation? It is know (Lindelöf) that $\mu(\sigma)$ is a convex function. Writing $\lambda_{0}$ for the abscissa of convergence

$$
\lambda_{0}=\inf \{\sigma \mid(1) \text { converges }\}
$$

we know that

$$
\mu\left(\lambda_{0}+1\right)=0
$$


because $f(s)$ is bounded when $\sigma>\lambda_{0}+1+\varepsilon(\varepsilon>0)$ and

$$
\mu\left(\lambda_{0}\right) \leq 1
$$

because of (3). Moreover (Schnee)

$$
\lambda_{0} \leq \inf \{\sigma \mid \mu(\sigma)=0\} .
$$

The first set of results of Bohr is of a rather negative character: (7), (8), (9) cannot be improved, which indicates a rather loose connection between convergence of (1) and order properties of $f(s)$.

THEOREM HB1. There exists a series (1) such that $\mu\left(\lambda_{0}\right)=0$ and $\mu(\sigma)<\infty$ for some $\sigma<\lambda_{0}$ (therefore, $\mu(\sigma)<\varepsilon$ does not imply convergence at $\sigma$ ) and a series (1) such that $\mu\left(\lambda_{0}\right)=1$ (therefore, convergence at $\sigma$ does not give anything better than Jensen's result).

This theorem (Theorems 16 and 17 of part I) concludes what Bohr calls "theory of convergence for Dirichlet series". No real progress was made on this theory-that is, the link between $\lambda_{0}$ and the properties of $f$-until $\mathbf{H}$. Helson gave (1962) a simple interpretation of $\lambda_{0}$ (when $\lambda_{0} \geq 0$ ) in terms of the Fourier properties of the functions

$$
\frac{f(\sigma+i t)}{\sigma+i t}
$$

as functions of the variable $t[6]$. Therefore, in the view of Bohr, there was no close structural link between convergence of (1) and properties of $f(s)$.

However, in part II of the thesis, entitled "the theory of summability for Dirichlet series”, Bohr discovers an essential link between order and summability. He introduces the Cesaro method of summability of order $k, C(k)$, and the absissas

$$
\left\{\begin{array}{l}
\lambda_{k}=\inf \{\sigma \mid(1) \text { is summable } C(k)\} \\
\lambda_{\infty}=\inf \lambda_{k}
\end{array} \quad(k=1,2, \ldots)\right.
$$

together with

$$
\mu_{\infty}=\inf \{\sigma \mid \mu(\sigma)<\infty\}
$$

with the convention that $\mu(\sigma)=\infty$ if $f$ has a singular point $s=\sigma+i$.

THEOREM HB2. $\lambda_{\infty}=\mu_{\infty}$.

In other words "a Dirichlet series $\sum a_{n} n^{-s}$ is summable just so far as the function $f(s)$ represented by the series is regular and of finite order of magnitude with respect to the ordinate $t$ " (this is the fundamental result of part II, cf. Section II.8).

It is possible to say more about the $\lambda_{k}$. Bohr proves the following. 
THEOREM HB3. (1) The $\lambda_{k}$ defined by (6) and (11) satisfy

$$
1 \geq \lambda_{k-1}-\lambda_{k} \geq \lambda_{k}-\lambda_{k-1} \geq \cdot \geq 0 \quad(k=1,2, \ldots) .
$$

(b) given any sequence $\lambda_{k}(k=0,1,2, \ldots)$ which satisfies (13), there exists a series (1) for which these are the abscissas of $C(k)$-summability (see (6) and (11)).

This is stated and proved in Section II.5.

It seems difficult to go much further. However Bohr was not completely satisfied. He know (as well as and independently of Marcel Riesz; see the footnote after Theorem 1 in Section II.8) that there is a close connection between the order function $\mu(\sigma)$ and the summability sequence $\lambda_{k}$. What is it exactly? He went back to the subject at the very end of his life. In the last paper published when he was alive [3] he introduces the "summability function" of $f, \psi(\sigma)$, defined in the natural way

$$
\begin{cases}\psi(\sigma)=0 & \text { when } \sigma \geq \lambda_{0} \\ \psi(\sigma)=k & \text { when } \lambda_{\infty}<\sigma=\lambda_{k} \leq \lambda_{0}\end{cases}
$$

where now $\lambda_{k}$ is defined as in (11), without the condition that $k$ is an integer (this generalization is due to $M$. Riesz). This function $\psi(\sigma)$ enjoys the following properties: it is convex with slope $\leq-1$ on $] \lambda_{\infty}, \lambda_{0}$ ] (in other words it is convex on $\left.] \lambda_{\infty}, \lambda_{0}\right]$ and

$$
\left.\psi^{\prime}\left(\lambda_{0}-0\right) \leq-1\right)
$$

and for all $\sigma>\lambda_{\infty}$

$$
\psi(\sigma) \leq \mu(\sigma) \leq \psi(\sigma)+1 .
$$

The Hauptsatz of [3] says that (16) cannot be improved.

TheOREM HB4. There exists a series (1) such that $\lambda_{\infty}=\mu_{\infty}=-\infty$ and

$$
\mu(\sigma)=\psi(\sigma)= \begin{cases}0 & \text { for } \sigma \leq 0, \\ -\sigma & \text { for } \sigma \leq 0 .\end{cases}
$$

There exists a series (1) such that $\lambda_{\infty}=\mu_{\infty}=-\infty$,

$$
\mu(\sigma)=\left\{\begin{array}{ll}
0 & \text { for } \sigma \geq 1, \\
1-\sigma & \text { for } \sigma \leq 1
\end{array} \text { and } \psi(\sigma)= \begin{cases}0 & \text { for } \sigma \geq 0, \\
-\sigma & \text { for } \sigma \leq 0 .\end{cases}\right.
$$

Actually the Hauptsatz of [3] is the second statement. The first was already proved (in a much easier way) in a previous paper [2]. At the end of [2] Bohr asks the following question. 
Problem HB5. Does there exist a series (1) with $\sigma_{c}$ (abscissa of convergence, also named $\left.\lambda_{0}\right)=0, \sigma_{A}$ (abscissa of absolute convergence) $=1$ and

$$
\mu(\sigma)=\frac{1}{2}-\sigma \text { when } 0<\sigma \leq \frac{1}{2} ?
$$

Bohr remarks that

$$
\sum_{1}^{\infty}(-1)^{n-1} n^{-s} \quad\left(=\zeta(s)\left(1-2^{1-s}\right)\right)
$$

provides an example if Lindelöf's hypothesis (that is, $\mu\left(\frac{1}{2}\right)=0$ for (20)) is true.

It happens that this problem is solved in an affirmative way in [8] (I was not aware of the problem in 1973), in a very simple manner as we shall see.

HB4 improves HB1. HB2 needs no improvement. Is it possible to improve HB3 by showing that the convexity of $\psi$ and $\mu$, together with (15) and (16), are characteristic properties of the couple constituted by the summability function and the order function? This was really the last problem which Bohr attacked. The main theorem of the posthumous paper [4] is the following (I quote it literally).

THEOREM HB6. Let $\psi(\sigma)$ be a continuous convex function defined in an interval $\sigma>\Omega(\geq-\infty)$ and equal to 0 to the right of a certain finite abscissa $\omega_{\psi} \geq \Omega$ and (if $\omega_{\psi}>\Omega$ ) such that $\psi^{\prime}\left(\omega_{\psi}-0\right) \leq-1$. Further, let $\mu(\sigma)$ be a continuous convex function defined in the same interval $\sigma>\Omega$ and equal to 0 to the right of a certain finite abscissa $\omega_{\mu} \geq \Omega$ and (if $\omega_{\mu}>\Omega$ ) such that $\mu^{\prime}\left(\omega_{\mu}-0\right) \leq-1$. Finally let $\psi(\sigma) \leq \mu(\sigma) \leq \psi(\sigma)+1$ for all $\sigma>\Omega$. Then there exists a Dirichlet series $f(s)=\sum a_{n} n^{-s}$ which has the given functions $\psi(\sigma)$ and $\mu(\sigma)$ as summability function and order function respectively.

At a first look it seems the end of the question: Bohr assumes nothing but the necessary conditions (15) and (16), together with the convexity of $\psi$ and $\mu$ and a small additional condition, namely

$$
\mu^{\prime}\left(\omega_{\mu}-0\right) \leq-1
$$

But is (21) as well as (15) a necessary condition? Here again I quote Bohr. Here comes the last problem.

"We remark that as a consequence of the assumptions of the theorem we have $\omega_{\psi} \leq \omega_{\mu} \leq \omega_{\psi}+1$. The condition $\omega_{\mu} \leq \omega_{\psi}+1$, which according to the above results is necessary whether $\mu^{\prime}\left(\omega_{\mu}-0\right) \leq-1$ or not, therefore has not been included in the theorem". And Bohr goes on: 
Problem HB7. "We do not know whether there exist ordinary Dirichlet series $f(s)=\sum a_{n} n^{-s}$ for which the order function $\mu(\sigma)$ is not identically zero and does not satisfy the condition $\mu^{\prime}\left(\omega_{\mu}-0\right) \leq-1$. For the zeta-series with alternating signs

$$
\zeta(s)\left(1-2^{1-s}\right)=\sum_{n=1}^{\infty}(-1)^{n+1} n^{-s}
$$

it is known that $\mu(\sigma)=0$ for $\sigma \geq 1$ and $\mu(\sigma)=\frac{1}{2}-\sigma$ for $\sigma \leq 0$. The question as to whether $\mu^{\prime}\left(\omega_{\mu}-1\right) \leq-1$ therefore amounts to whether $\mu\left(\frac{1}{2}\right)=0$ (and hence $\mu(\sigma)=0$ for $\sigma \geq \frac{1}{2}$ and $\mu(\sigma)=\frac{1}{2}-\sigma$ for $\left.\sigma \leq \frac{1}{2}\right)$, that is, to the Lindelö hypothesis $\zeta\left(\frac{1}{2}+i t\right)=O\left(|t|^{\varepsilon}\right)$ for every $\varepsilon>0$."

This seemed a very challenging question to me. Since (21) can be written as

$$
\mu(\sigma+\alpha) \leq \sup (0, \mu(\sigma)-\alpha)
$$

for all $\sigma$ and $\alpha>0$, I tried to get sharp upper estimates for $\mu(\sigma+\alpha)$ given $\mu(\sigma)$ and $\alpha$. The best I can prove is

$$
\mu\left(\sigma+\mu(\sigma)+\frac{1}{2}\right)=0
$$

and what can be derived from (23) by convexity arguments. Then I found an example with $\mu^{\prime}\left(\omega_{\mu}-0\right)>-1$, and I can go as far as

$$
\mu^{\prime}\left(\omega_{\mu}-0\right)>-\frac{1}{2}-\varepsilon \quad(\varepsilon>0 \text { given }) .
$$

This will be the content of section III.

Though this can be considered as a solution for HB7, we are now in a difficult situation and the problem remains as open as ever. On one hand, there is no way to derive Lindelöfs hypothesis from a general result (namely, that (21) holds for all series (1) such that $\mu(\sigma)$ is not identically 0 ). On the other hand, we are far from necessary and sufficient conditions on two convex functions to be the summability function and the order function respectively of some ordinary Dirichlet series. It seems already quite difficult-and challenging - to get a characterization of the order functions $\mu(\sigma)$ of ordinary Dirichlet series; translated in this way, the last problem of Bohr remains open.

\section{A way to answer HB5 and to perform Bohr's constructions}

(after [8] and [11])

Let us look at series (2) in the form

$$
\sum_{1}^{\infty} \varepsilon_{n} n^{-s}
$$


where $\varepsilon=\left(\varepsilon_{1}, \varepsilon_{2}, \ldots\right) \in\{-1,1\}^{\mathcal{N}^{+}}=\Omega$. We can consider $\Omega$ as a probability space (provided with the natural probability: $P\left(\varepsilon_{n}=1\right)=P\left(\varepsilon_{n}=-1\right)=\frac{1}{2}$ and the $\varepsilon_{n}$ are independent); therefore we can speak of almost sure properties of (25). We can also consider $\Omega$ as a topological space, metric and compact; we shall say that (25) has a quasisure property $\mathscr{P}$ if $\mathscr{P}$ occurs when $\varepsilon$ belongs to some dense $G_{\delta}$-set (a set of the second category in the sense of Baire). Here is an easy result about the abscissa of convergence $\lambda_{0}$, the order function $\mu(\sigma)$ and the analytical continuation of the function $f_{\varepsilon}(s)$ represented by $(25)$.

THEOREM 2.1. Almost surely for series (25) we have

$$
\left\{\begin{array}{l}
\lambda_{0}=\frac{1}{2} \\
\mu(\sigma)=0 \text { for } \sigma>\frac{1}{2}
\end{array}\right.
$$

and the line $\sigma=\frac{1}{2}$ is a natural boundary. Quasi-surely we have

$$
\left\{\begin{array}{l}
\lambda_{0}=1 \\
\mu(\sigma)=0 \text { for } \sigma>1
\end{array}\right.
$$

and the line $\sigma=1$ is a natural boundary.

To go further we consider series (2) of the form

$$
\sum_{1}^{\infty} \varepsilon_{n}\left((2 n-1)^{-s}-(2 n)^{-s}\right)
$$

where $\varepsilon \in \Omega$ as before. Then we obtain

THEOREM 2.2. Almost surely for series (28) we have

$$
\left\{\begin{array}{l}
\lambda_{0}=0 \\
\mu(\sigma)=\sup \left(\frac{1}{2}-\sigma, 0\right) \quad \text { for } \sigma>-\frac{1}{2}
\end{array}\right.
$$

and the line $\sigma=-\frac{1}{2}$ is a natural boundary. Quasi-surely we have

$$
\left\{\begin{array}{l}
\lambda_{0}=0 \\
\mu(\sigma)=\sup (1-\sigma, 0) \text { for } \sigma>0
\end{array}\right.
$$

and the line $\sigma=0$ is a natural boundary.

Theorem 2.2 is not difficult (see [8] or [11]). The first part answers HB5. The second part provides the second statement in HB1 (the difficult part in Bohr's approach). The first statement of HB1 needs series of the form

$$
\sum_{1}^{\infty} \delta_{n}\left((2 n-1)^{-s}-(2 n)^{-s}\right)
$$


where $\delta_{n}=0$ or 1 and (31) is very lacunary (for example,

$$
\left.\sum_{1}^{\infty} \delta_{n} \log \log \frac{1}{n}<\infty\right)
$$

Then (assuming also $\sum \delta_{n}=\infty$ )

$$
\left\{\begin{array}{l}
\lambda_{0}=0 \\
\mu(\sigma)=\sup (-\sigma, 0) \text { for } \sigma>-1
\end{array}\right.
$$

and the line $\sigma=-1$ is a natural boundary. This is Bohr's construction.

We can go further and consider series

$$
\sum_{1}^{\infty} \varepsilon_{n}\left((4 n-3)^{-s}-(4 n-2)^{-s}-(4 n-1)^{-s}+(4 n)^{-s}\right)
$$

instead of (28) and

$$
\sum_{1}^{\infty} \delta_{n}\left((4 n-3)^{-s}-(4 n-2)^{-s}-(4 n-1)^{-s}+(4 n)^{-s}\right)
$$

instead of (31). The results are easy to guess (and also to prove): we have $\lambda_{0}=0$ as before and the same expression for $\mu(\sigma)$, but now the range is extended, that is, $\sigma>-\frac{3}{2}$ (and the line $\sigma=-\frac{3}{2}$ is a natural boundary) a.s. for (34), $\sigma>-1$ (and the line $\sigma=-1$ is a natural boundary) q.s. for (34), $\sigma>-2$ (and the line $\sigma=-2$ is a natural boundary) for (35).

If we want to get entire functions we need differences of higher and higher order. This is what Bohr does in [2] for lacunary series, and what Queffelec performs in [11] for series (2) with a.s. or q.s. properties. Let me use Queffelec's notations. For each $\phi \in C^{\infty}[1, \infty)$ let us write

$$
\begin{aligned}
& \Delta_{0} \phi(n)=\phi(n) \quad(n=1,2, \ldots) \\
& \Delta_{1} \phi(n)=\phi(n+1)-\phi(n)=\int_{0}^{1} \phi^{\prime}\left(n+x_{1}\right) d x_{1} \\
& \Delta_{2} \phi(n)=\Delta_{1} \phi(n+2)-\Delta_{1} \phi(n)=\iint_{[0,1] \times[0,2]} \phi^{\prime \prime}\left(n+x_{1}+x_{2}\right) d x_{1} d x
\end{aligned}
$$

$$
\begin{aligned}
\Delta_{p} \phi(n) & =\Delta_{p-1} \phi\left(n+2^{p-1}\right)-\Delta_{p-1} \phi(n) \\
& =\iint_{[0,1] \times[0,2] \cdots\left[0,2^{p-1}\right]} \phi^{(p)}\left(n+x_{1}+\cdots+x_{p}\right) d x_{1} \cdots d x_{p}
\end{aligned}
$$

Formula (36) allows sharp estimates for $\Delta_{p} \phi(n)$ when we choose $\phi(x)=x^{-s}$ ( $s$ complex). From now on we choose

$$
\left\{\begin{array}{l}
\phi(x)=x^{-s} \\
n_{j}=\left(j^{2}\right) ! \quad(j=0,1, \ldots)
\end{array}\right.
$$


(many other choices of lacunary sequences are possible) and we define positive integers $u_{j n}\left(n_{j}<n \leq n_{j+1}, j=0,1, \ldots\right)$ in such a way that the series

$$
\sum_{j=0}^{\infty}\left(\sum_{n_{j}<n \leq n_{j+1}} \varepsilon_{j n} \Delta_{j} \phi\left(u_{j n}\right)\right) \quad\left(\varepsilon_{j n}= \pm 1\right)
$$

has the form (2) (that is, each $m^{-s}$ can be found once and once only among the $\left.\Delta_{j} \phi\left(u_{j n}\right)\right)$. Here again $\varepsilon=\left(\varepsilon_{j n}\right) \in \Omega$.

Theorem 2.3. Almost surely for series (38) the function $f_{\varepsilon}(s)$ is an entire function and we have

$$
\left\{\begin{array}{l}
\lambda_{0}=0 \\
\mu(\sigma)=\sup \left(\frac{1}{2}-\sigma, 0\right) \quad(\sigma \in \mathscr{R}) .
\end{array}\right.
$$

Quasi-surely the function $f_{\ell}(s)$ is an entire function and we have

$$
\left\{\begin{array}{l}
\lambda_{0}=0 \\
\mu(\sigma)=\sup (1-\sigma, 0) \quad(\sigma \in \mathscr{R})
\end{array}\right.
$$

For the proof see [11]. (39) is the expected behaviour of the series (20), $\sum(-1)^{n+1} n^{-s}$, by Lindelöf's hypothesis. Therefore the first part of the Theorem 2.3 provides a nice answer to the problem HB5: there exist entire functions represented by series (2) whose order and summability functions are those expected for $\zeta(s)\left(1-2^{1-s}\right)$.

The second part of Theorem 2.3 provides the Hauptsatz of [3], that is, the second half of HB4. For the first part of HB4 a lacunary series of the form

$$
\sum_{1}^{\infty} \delta_{n} \Delta_{n} \phi(n) \quad\left(\delta_{n}=0 \text { or } 1\right)
$$

is convenient, and this is essentially what Bohr uses in [2].

Let me indicate how the method of Theorem 2.3 can be used in order to obtain Theorem HB6. For simplicity I shall restrict myself to the case $\Omega=-\infty$ (entire functions) and $\phi^{\prime}$ and $\mu^{\prime}$ have only integral values. Then we can write

$$
\begin{cases}\phi(\sigma)=\sup \phi_{k}(\sigma) & (k=1,2, \ldots) \\ \mu(\sigma)=\sup \mu_{k}(\sigma) & (k=1,2, \ldots)\end{cases}
$$

where both $\phi_{k}(\sigma)$ and $\mu_{k}(\sigma)$ vanish when $\sigma$ is large and are linear with slope $-k$ as soon as they are not 0 , that is

$$
\left\{\begin{array}{l}
\phi_{k}(\sigma)=\sup \left(-k\left(\sigma-\omega_{k}\right), 0\right) \\
\mu(\sigma)=\sup \left(-k\left(\sigma-\omega_{k}^{\prime}\right), 0\right)
\end{array}\right.
$$


and moreover

$$
\phi_{k}(\sigma) \leq \mu_{k}(\sigma) \leq \phi_{k}(\sigma)+1
$$

or, equivalently

$$
\omega_{k} \leq \omega_{k}^{\prime} \leq \omega_{k}+1 / k
$$

If we are given $\phi_{k}$ and $\mu_{k}$ with these properties, Theorem 2.3 provides very easily a Dirichlet series (1) for which they are the summability function and the order function respectively. We remark that $\omega_{k}$ has to be the abscissa of convergence, and we can suppose $\omega_{k}=0$ without restriction. Let us consider the case $k=1$ first. There are two extreme cases: (a) $\omega_{k}^{\prime}=\omega_{k}$; (b) $\omega_{k}^{\prime}=\omega_{k}+1$. (41) provides the desired series in case (a) (note that the summability function has a slope $\leq-1$ when $\sigma<\omega_{k}$ and satisfies (16), therefore it coincides with the order function) and quasi-surely (38) gives the desired series in case (b). We do not use the almost sure properties of (38). When we are in between the extreme cases, we simply solve the problem for $\left(\omega_{k}, \omega_{k}\right),\left(\omega_{k}^{\prime}-1, \omega_{k}^{\prime}\right)$, and add the solutions. This solves the case $k=1$. Now we observe that, given an ordinary Dirichlet series (B1), if we change $s$ into $k s$, we get another Dirichlet series, whose summability and order functions are $\mu(k \sigma)$ and $\phi(k \sigma)$ respectively. This solves the general case.

Suppose now that we are given $\psi(\sigma)$ and $\mu(\sigma)$ as in (42), where the $\psi_{k}(\sigma)$ and $\mu_{k}(\sigma)$ satisfy (43) and (44). The idea is just to add the Dirichlet series corresponding to $\left(\psi_{k}, \mu_{k}\right)$. However, it is necessary either to get uniform estimates, or to proceed by induction, removing from the $k$ th Dirichlet series a number of terms sufficiently large, not to perturb the effect of the previous Dirichlet series on $\psi(\sigma)$ and $\mu(\sigma)$. I shall not give the details; let me just observe that we used lacunary series (41) and the quasi-sure properties of (38) only. Moreover, we converted (38) into lacunary series by changing $s$ into $k s$.

When $\psi(\sigma)$ and $\mu(\sigma)$ do not enjoy the property above, that $\psi^{\prime}$ and $\mu^{\prime}$ have only integral values, we still write (42), but now for real $k \geq 1$. In this case we have to construct lacunary series without using the trick of changing $s$ into $k s$. There is not difficulty for series (41). For series (38) we have to give up the condition that it gives series (2), and again there is no essential difficulty.

The idea of reducing the question on $(\psi, \mu)$ to the question of $\left(\psi_{k}, \mu_{k}\right)$, and then to $\left(\psi_{k}, \mu_{k}\right)$ in the extreme cases (that is, one or the other equality in (45)) is exactly Bohr's idea. He says that the solutions given $\left(\psi_{k}, \mu_{k}\right)$ in the extreme cases are the "building blocks" of the general solution. He considers that one of the extreme cases $\left(\omega_{k}=\omega_{k}^{\prime}\right)$ is relatively easy, and he spends the largest part of his paper [4] in building the other blocks $\left(\omega_{k}^{\prime}=\omega_{k}+1 / k\right)$. The use of Baire arguments is just a simplification in order to get these building blocks. 


\section{The Lindelöf function for ordinary Dirichlet series}

Here we consider the last problem of Harald Bohr (HB7). What can we say about $\mu(\sigma)$ ? I have already stated the main results at the end of Section 1. In the first part of this section I shall prove (23), and in the second part exhibit an example which satisfies (24).

First we shall try to prove

$$
\mu(\sigma+\alpha) \leq \sup (0, \mu(\sigma)-\alpha+\varepsilon)
$$

for all series (1), $\sigma \in \mathscr{R}, \alpha>0$ and some $\varepsilon=\varepsilon(\mu(\sigma), \alpha)$. It is enough to consider finite sums

$$
f(s)=\sum a_{n} n^{-s}
$$

and to prove that the condition

$$
\forall t \in \mathscr{R} \quad|f(\sigma+i t)| \leq \sup \left(1,|t|^{\beta}\right)
$$

implies that for all $\theta>1$

$$
|f(\sigma+\alpha+i \theta)| \leq C \theta^{\gamma}
$$

with $\gamma=\gamma(\alpha, \beta) \geq 0$ and $C=C(\alpha, \beta, \gamma)$. Then (46) holds with $\beta-\alpha+\varepsilon=\gamma$. Moreover we can assume $\sigma=0$ without any restriction.

The implication (48) implies (49) is equivalent to the existence of a complex measure $d \mu_{\theta}(t)$ on $\mathscr{R}$ such that

$$
\left\{\begin{array}{l}
n^{-\alpha-i \theta}=\int n^{-i t} d \mu_{\theta}(t) \quad(n=1,2, \ldots) \\
\int \sup \left(1,|t|^{\beta}\right)\left|d \mu_{\theta}(t)\right| \leq C \theta^{\gamma}
\end{array}\right.
$$

Let us introduce the Fourier transform

$$
\hat{\mu}_{\theta}(x)=\int e^{-i t x} d \mu(t)
$$

and the space $B(\beta)$ which consists of Fourier transforms $\hat{\mu}$ of measures $d \mu$ such that $|d \mu|$ integrates $\sup \left(1, \mid t^{\beta}\right)$, with the norm

$$
\|\hat{\mu}\|_{B(\beta)}=\int \sup \left(1,|t|^{\beta}\right)|d \mu(t)| .
$$

It is known and very easy to check that $B(\beta)$ is a Banach algebra with pointwise multiplication. Given $F$, any closed subset of $\mathscr{R}$, we write $B(\beta, F)$ for the quotient algebra of $B(\beta)$ by the ideal of functions which vanish on $F$, with the norm

$$
\|p\|_{B(\beta, F)}=\inf \left\{\|\hat{\mu}\|_{B(\beta)} \mid \hat{\mu}=p \text { on } F\right\} .
$$

Let us write

$$
\Lambda=\{\log n\} \quad(n=1,2, \ldots) .
$$


Then the existence of $d \mu_{\theta}$ with (50) is equivalent to

$$
\left\|e^{-(\alpha+i \theta) x}\right\|_{B(\beta, \Lambda)} \leq C \theta^{\gamma} .
$$

Let us consider the case $\beta<1$ first. It is easier because in this case $e^{-\alpha|x|}$ belongs to $B(\beta)$. Given $a>0$, let us write

$$
\left\{\begin{array}{l}
p(x)=e^{-(\alpha+i \theta) x} \\
q(x)=e^{-\alpha a} e^{-\alpha|x-a|} e^{-i \theta x}
\end{array}\right.
$$

Then we have (note that the norm of $e^{-i \theta x}$ is $\theta^{\beta}$ )

$$
\left\{\begin{array}{l}
p(x)=q(x) \text { for } x \geq a \\
\|q\|_{B(\theta)} \leq C \theta^{\beta} e^{-\alpha a} \quad(C=C(\alpha, \beta))
\end{array}\right.
$$

Writing

$$
r(x)=p(x)-q(x)=\left\{\begin{array}{l}
0 \text { on }(a, \infty) \\
\left(e^{-\alpha x}-e^{\alpha(x-2 a)}\right) e^{-i \theta x} \text { on }(0, a)
\end{array}\right.
$$

we want to estimate $\|r\|_{B(\beta, \Lambda)}$.

Let us suppose now that

$$
\left\{\begin{array}{l}
a=\log (N+1) \\
N=N(\theta, \beta)=2^{l}-1
\end{array}\right.
$$

$N(\theta, \beta)$ will be defined later. We are given

$$
r_{n}=r(\log n)
$$

so that $\left|r_{n}\right| \leq n^{-\alpha}$ for $n=1,2, \ldots N$ and $r_{n}=0$ for $n>N$. Given an integer $\nu<N / 2$ we consider

$$
r(x, \nu)=\sum_{\nu \leq n<2 \nu} r_{n} \Delta(\nu(x-\log n))
$$

where $\Delta$ is a $C^{\infty}$ function carried by the interval $\left[-\frac{1}{2}, \frac{1}{2}\right]$ such that $\Delta(0)=1$. Since

$$
\log (n+1)-\log n \geq 1 /(n+1)>1 / 2 \nu
$$

when $\nu \leq n<2 \nu$, the restriction of $r(\cdot, \nu)$ to $\Lambda$ coincides with the given $\left.r\right|_{\Lambda}$ on the interval $[\log \nu, \log (2 \nu-1)]$ and vanishes on the rest of $\Lambda$. A good candidate for an extrapolation of $\left.r\right|_{\Lambda}$ on $\mathscr{R}$ is therefore

$$
r(x)=\sum_{j=0}^{l-1} r\left(x, 2^{j}\right) \text {. }
$$

Let us estimate the norm of $r(\cdot, \nu)$ in $B(\beta)$. A very crude estimate is

$$
\begin{aligned}
\|r(\cdot, \nu)\|_{B(\beta)} & \leq \|\left(\Delta(\nu \cdot) \|_{B(\beta)} \sum_{\nu \leq n<2 \nu} n^{-\alpha}\right. \\
& =O\left(\nu^{\beta-\alpha+1}\right) .
\end{aligned}
$$


Then, adding, we obtain

$$
\|r\|_{B(\beta)}=O\left(N^{\beta-\alpha+1}\right)
$$

whenever $\beta-\alpha+1>0$. Hence, using

$$
\|p\|_{B(\beta, \Lambda)} \leq\|q\|_{B(\beta)}+\|r\|_{B(\beta)}
$$

we obtain

$$
\|p\|_{B(\beta, \Lambda)} \leq C\left(\theta^{\beta} N^{-\alpha}+N^{\beta-\alpha+1}\right)
$$

and choosing

$$
N^{\beta+1} \simeq \theta^{\beta}
$$

(this is how we define $N(\theta, \beta)$ in (59)) we get (55) with

$$
\gamma=\beta-\alpha \beta /(\beta+1) \text {. }
$$

Any improvement of (65) gives an improvement of (69). Here is now the best I can do. Writing $\hat{\Delta}$ for the Fourier transform of $\Delta$ we have

$$
\begin{aligned}
\|r(\cdot, \nu)\|_{B(\beta)} & =\int\left|\sum_{\nu \leq n<2 \nu} r_{n} e^{i t \log n} \nu^{-1} \hat{\Delta}\left(\nu^{-1} t\right)\right| \sup \left(1,|t|^{\beta}\right) d t \\
& =\sum_{k \in \mathcal{I}} \int_{k \nu}^{(k+1) \nu} \text { idem } \\
& \leq C \nu^{\beta} \sup _{k} \frac{1}{\nu} \int_{k \nu}^{(k+1) \nu}\left|\sum_{\nu \leq n<2 \nu} r_{n} e^{i t \log n}\right| d t .
\end{aligned}
$$

Now

$$
\frac{1}{\nu} \int_{\nu}^{(k+1) \nu} \| d t \leq\left(\frac{1}{\nu} \int_{k \nu}^{(k+1) \nu} \|^{2} d t\right)^{1 / 2}
$$

and a well known inequality of Ingham either in its original form or in the refined version of Montgomery and Vaughan [10] says that the second member in (71) is $O\left(\left(\sum r_{n}^{2}\right)^{1 / 2}\right)$, that is in our case, $O\left(\nu^{-\alpha+1 / 2}\right)$. Then, instead of (65), we have

$$
\|r\|_{B(\beta)}=O\left(N^{\beta-\alpha+1 / 2}\right)
$$

whenever $\beta-\alpha+1 / 2>0$. Therefore we get (55) with

$$
\gamma=\beta-2 \alpha \beta /(2 \beta+1)
$$

instead of (69).

In the limit case $\alpha=\beta+1 / 2$ we have to write

$$
\|r\|_{B(\beta)}=O(\log N)
$$


instead of (72). We may choose $N \bar{\theta} \theta$ and we obtain

$$
\|p\|_{B(\beta, \Lambda)}=O(\log \theta) .
$$

Let us state the results (the case $\beta \geq 1$ needs only minor changes).

THEOREM 3.1. If $f(s)$ is either the sum of an ordinary Dirichlet series or its analytic continuation in a vertical halfplane containing $\sigma_{0}$ and if

$$
f\left(\sigma_{0}+i t\right)=O\left(|t|^{\beta}\right) \quad(|t| \rightarrow \infty)
$$

then

$$
f\left(\sigma_{0}+\beta+\frac{1}{2}+i \theta\right)=O((|\log \theta|)(|\log \theta|) \rightarrow \infty) .
$$

COROLLARY. The inequality

$$
\mu\left(\sigma+\mu(\sigma)+\frac{1}{2}\right) \geq 0
$$

holds for all Lindelof functions of ordinary Dirichlet series. Equivalently, the inequality

$$
\mu(\sigma) \geq \sup \left(\omega_{\mu}-\frac{1}{2}-\sigma, 0\right)
$$

holds, if we define

$$
\omega_{\mu}=\inf \{\sigma \mid \mu(\sigma)=0\}
$$

This is the best information which the method provides. The estimate for $\mu(\sigma+\alpha)$ which derives from (78) by convexity is exactly what (73) gives.

Let me add two remarks. First, (62) is the only property of the sequence $\Lambda$ which we have used. Therefore, Theorem 3.1 is valid for "general" Dirichlet series

$$
\sum a_{n} e^{-\lambda_{n} s}
$$

whenever

$$
\lambda_{n+1}-\lambda_{n} \geq 1 / n+1 \quad(n=1,2, \ldots)
$$

holds. For this generalized version of Theorem 3.1 it is easy to see that $\frac{1}{2}$ in (78) or (79) cannot be replaced by any smaller constant.

Here is the second remark. Since the crucial point in the proof of Theorem 3.1 is the uniform estimate

$$
\frac{1}{\nu} \int_{k \nu}^{(k+1) \nu}\left|\sum_{\nu \leq n<2 \nu} r_{n} e^{i t \log n}\right| d t=O\left(\nu^{1 / 2-\alpha}\right)
$$


which derives easily from

$$
\frac{1}{\nu} \int_{k \nu}^{(k+1) \nu}\left|\sum_{\nu \leq n<2 \nu} e^{-i \theta \log n} e^{i t \log n}\right| d t=O\left(\nu^{1 / 2}\right)
$$

it is tempting to consider the apparently simpler question: is it true that

$$
\frac{1}{\nu} \int_{0}^{\nu}\left|\sum_{n=1}^{\mu} e^{i t \log n}\right| d t=O\left(\nu^{1 / 2-\varepsilon}\right) \quad(1 \leq \mu \leq \nu)
$$

for some $\varepsilon>0$ ? The answer is no. For, assuming (85), consider

$$
R_{\nu}(x)=\sum_{n=1}^{\nu} \Delta(\nu(x-\log 2 n))
$$

where $\Delta$ has the same meaning as in (61). Then

$$
\left\|R_{\nu}\right\|_{B(\beta)}=O\left(\nu^{\beta+1 / 2-\varepsilon}\right) .
$$

Note that $R_{\nu}(\log m)=1$ when $m$ is even between 2 and $2 \nu$, and $R_{\nu}(\log m)=0$ for all other values of $m$. Let $B^{*}(\beta)$ be the dual of $B(\beta)$. Since $\mu(0)=\frac{1}{2}$ for the function (20) we have

$$
T_{\sigma}=\sum_{1}^{\infty} m^{-\sigma}(-1)^{m} \delta_{\log m} \in B^{*}\left(\frac{1}{2}\right)
$$

for each $\sigma>0$. Now

$$
\left(T_{\sigma}, R_{\nu}\right)=\sum_{n=1}^{\nu}(2 n)^{-\sigma} \leq\left\|T_{\sigma}\right\|_{B^{*}(\beta)}\left\|R_{\nu}\right\|_{B(\beta)}
$$

and choosing $\beta=\frac{1}{2}$ and $\sigma<\varepsilon$ (89) contradicts (87).

Let us look now in the opposite direction. Our purpose is to construct a series (1) such that the corresponding Lindelöf function $\mu(\sigma)$ has somewhere a slope strictly between -1 and 0 , or, equivalently,

$$
\mu^{\prime}\left(\omega_{\mu}-0\right)>-1 .
$$

In a first step we shall construct a series (1) such that

$$
\mu(0)=1, \quad \mu(1)>0 .
$$

This is sufficient to solve the question HB7 stricto sensu.

In a second step, given $0<\beta<1$, we construct a series (1) such that

$$
\mu(0)=\beta, \quad \mu(\beta)>0
$$

and (24) holds (when we are given $\beta=\beta(\varepsilon)$ small enough).

We shall use the following tool ([9], Lemma 3). It exhibits a trigonometric polynomial whose $L^{\infty}$-norm is near the $L^{2}$-norm, and much smaller than the $l^{1}$-norm of the coefficients. Many variations are possible. However the special arguments of the coefficients will prove useful. 
LEMMA. Let $a$ be an even positive integer, $0<d<\frac{1}{3}$, $\phi_{0}$ a function with bounded variation $V$ carried by the interval $[-(1-3 d) / 2,(1-3 d) / 2], \chi_{d}$ the indicator function of $[-d / 2, d / 2], \phi=d^{-3} \phi_{0} * \chi_{d} * \chi_{d} * \chi_{d}$,

$$
\begin{aligned}
\gamma_{m} & =\frac{1}{\sqrt{a}} \exp \left(-\pi i m^{2} / a\right) \phi(m / a), \\
\gamma(x) & =\sum_{m} \gamma_{m} e^{2 \pi i m x} .
\end{aligned}
$$

Then, for $|x| \leq \frac{1}{2}$,

$$
\gamma(x)=\exp \left(-\frac{\pi i}{4}\right) \exp \left(\pi i a x^{2}\right) \phi(x)+r(x), \quad|r(x)| \leq \frac{V}{2 \pi a d^{2}} .
$$

Actually we shall choose $\phi_{0}=1_{[-l, l]}$, consider that $d$ and $l$ are fixed, and use only $|\gamma(x)| \leq 2$. Let us observe that $\gamma_{m}=0$ when $|m| \geq\left(l+\frac{3 d}{2}\right) a$.

Let us introduce

$$
g(x)=\gamma(x)\left(1-e^{\pi i x}\right)=\sum c_{m} e^{\pi i m x} .
$$

Then we have $c_{2 m}=-c_{2 m+1}=\gamma_{m}$ and

$$
|g(x)| \leq \inf (2 \pi|x|, 4) .
$$

The building block of the construction is the Dirichlet polynomial

$$
f(s)=f(s, N, a)=\sum c_{m}(N+m)^{-s}
$$

where $N$ is an integer (large with respect to $a$ ) to be defined later. It proves convenient to write

$$
\begin{aligned}
N^{-i t} f(i t) & =\sum c_{m} e^{-i t \log (1+m / N)} \\
& =\sum c_{m} e^{-i t\left(m / N+\varepsilon_{m} m^{2} / N^{2}\right)} \quad\left(\left|\varepsilon_{m}\right| \leq 1\right) \\
& =g\left(-\frac{t}{\pi N}\right)+r(t)
\end{aligned}
$$

where

$$
|r(t)| \leq \inf \left(\sum\left|c_{m}\right||t| \frac{m^{2}}{N^{2}}, \sum\left|c_{m}\right|\right)
$$

First step. We choose

$$
N=a^{5 / 2}+O(a)
$$

so that

$$
\sum\left|c_{m}\right| \frac{m^{2}}{N^{2}}=O\left(a^{5 / 2} N^{-2}\right)=O\left(N^{-1}\right)
$$


From (94), (96), (97), (99) we get

$$
f(i t)=O\left(\frac{|t|}{N}\right)
$$

(here $O(X)$ stands for bounded by some constant multiple of $X$ ).

Let us consider now $f(1+i \theta)$. We write

$$
N^{i \theta} f(1+i \theta)=\sum \frac{c_{m}}{N+m} e^{-i \theta\left(m / N-m^{2} / 2 N^{2}+\varepsilon_{m}^{\prime}|m|^{3} / N^{3}\right)}\left(\left|\varepsilon_{m}^{\prime}\right| \leq 1\right) .
$$

We assume, as we can, that

$$
N=2 a \times \text { odd integer }
$$

and choose

$$
\theta=\pi N^{2} / 2 a
$$

so that

$$
e^{-i \theta\left(m / N-m^{2} / 2 N^{2}\right)}=(-1)^{m} e^{\pi i m^{2} / 4 a} .
$$

Since

$$
\frac{c_{m}}{\left|c_{m}\right|}= \begin{cases}e^{\pi i m^{2} / 4 a} & (m \text { even }) \\ -e^{-\pi i(m-1)^{2} / 4 a} & (m \text { odd })\end{cases}
$$

we have

$$
\frac{c_{m}}{N+m} e^{-i \theta\left(m / N-m^{2} / 2 N^{2}\right)}=\frac{\left|c_{m}\right|}{N+m}=\frac{\left|c_{m}\right|}{N}\left(1+O\left(\frac{a}{N}\right)\right)
$$

when $m$ is even, and

$$
\operatorname{Re}\left(\frac{c_{m}}{N+m} e^{-i \theta\left(m / N-m^{2} / 2 N^{2}\right)}\right)>\left(\frac{1}{2}+O\left(\frac{1}{a}\right)\right) \frac{\left|c_{m}\right|}{N}
$$

when $m$ is odd and moreover we assume

$$
l+\frac{3 d}{2} \leq \frac{1}{3}
$$

Therefore

$$
\operatorname{Re}\left(\sum \frac{c_{m}}{N+m} e^{-i \theta\left(m / N-m^{2} / 2 N^{2}\right)}\right)>\left(\frac{1}{2}+O\left(\frac{1}{a}\right)\right) \frac{1}{N} \sum\left|c_{m}\right| .
$$

Now

$$
\left|N^{i \theta} f(1+i \theta)-\sum \frac{c_{m}}{N+m} e^{-i \theta\left(m / N-m^{2} / 2 N^{2}\right)}\right| \leq \frac{\theta}{N} \sum\left|c_{m}\right| \frac{|m|^{3}}{N^{3}}\left(1+O\left(\frac{a}{N}\right)\right) .
$$

Since

$$
\theta \frac{|m|^{3}}{N^{3}} \leq \theta \frac{a^{3}}{N^{3}}=O\left(N^{-1} a^{2}\right)=o(1)
$$

we obtain

$$
\operatorname{Re}\left(N^{i \theta} f(1+i \theta)\right)>\left(\frac{1}{8}+o(1)\right) \frac{1}{N} \sum\left|c_{m}\right|>C \frac{a^{1 / 2}}{N}
$$


because, due to the choice of $\phi$ in the lemma, we have

$$
\sum\left|c_{m}\right| \geq C \sqrt{a}
$$

( $C$ depending only on $d, l$, and the line where it is written). Since $a \sim N^{1 / 5}$ and $\theta \sim N^{8 / 5}$ we finally get

$$
\operatorname{Re}\left(N^{i \theta} f(1+i \theta)\right)>\frac{C}{N} \theta^{1 / 8} .
$$

Here $N f(s)=N f(s, N, a)$ is only a building block, which satisfies (100) and (105). Clearly by adding such blocks $j^{-2} N_{j} f\left(s, N_{j}, a_{j}\right)$ for a lacunary sequence $N_{j}$ we obtain a Dirichlet series for which $\mu(0) \leq 1$ (actually, $\mu(0)=$ $1)$ and $\mu(1) \geq \frac{1}{8}$. Let us state the result of this first step.

THEOREM 3.2. There exists a Dirichlet series for which the Lindeloff function has a derivative which satisfies $0>\mu^{\prime}(\sigma)>-1$ at some points $\sigma$ (in particular, $\left.\mu^{\prime}\left(\omega_{\mu}-0\right)>-1\right)$.

Our construction gives actually $\mu^{\prime}(\sigma) \geq-\frac{7}{8}$ at some $\left.\sigma \in\right] 0,1$, therefore

$$
\mu^{\prime}\left(\omega_{\mu}-0\right) \geq-\frac{7}{8} \text {. }
$$

It is very easy to improve (106) by considering $f(\alpha+i \theta)$ instead of $f(1+i \theta)$, with $f(s)=f(s, N, a)$ as before. We have now

$$
\operatorname{Re}\left(N^{i \theta} f(\alpha+i \theta)\right)>C N^{-\alpha} a^{1 / 2}
$$

therefore

$$
\operatorname{Re}\left(N^{i \theta} f(\alpha+i \theta)\right)>\frac{C}{N} \theta^{(6-5 \alpha) / 8} .
$$

In our example (sum of $j^{-2} N_{j} f\left(s, N_{j}, a_{j}\right)$ we obtain

$$
\mu(\alpha) \geq(6-5 \alpha) / 8
$$

therefore

$$
\omega_{\mu} \geq \frac{6}{5}, \quad \mu^{\prime}\left(\omega_{\mu}-0\right) \geq-\frac{6}{5} .
$$

SECOND STEP. We consider now $0<\beta<1$ and we intend to construct a series (1) for which $\mu(0) \leq \beta$ and $\mu(\alpha)$ is minorized by a convenient function of $\alpha$ and $\beta$. Now we choose

$$
N=a^{(4 \beta+1) / 2 \beta}+O(a)
$$

instead of (98) and we try to take advantage of the full force of (94) and (97), using $\inf (|x|, 1) \leq x^{\beta}$. We obtain

$$
\begin{aligned}
& \left|g\left(-\frac{t}{\pi N}\right)\right| \leq C\left(\frac{|t|}{N}\right)^{\beta} \\
& |r(t)| \leq C \sqrt{a}\left(\frac{|t| a^{2}}{N^{2}}\right)^{\beta}
\end{aligned}
$$


and our choice of $N$ gives equal weight to the second members. Then.

$$
f(i t)=O\left(N^{-\beta}|t|^{\beta}\right) \text {. }
$$

Again

$$
\operatorname{Re}\left(N^{i \theta} f(\alpha+i \theta)\right) \geq C a^{1 / 2} N^{-\alpha}
$$

and now

$$
a \sim N^{2 \beta /(4 \beta+1)}, \quad \theta \sim \frac{N^{2}}{a} \sim N^{(6 \beta+2) /(4 \beta+1)}
$$

so that

$$
\begin{aligned}
\operatorname{Re}\left(N^{i \theta} f(\alpha+i \theta)\right) & \geq C N^{-\beta} N^{\beta-\alpha+\beta /(4 \beta+1)} \\
& \geq C N^{-\beta} \theta^{((4 \beta+1) /(6 \beta+2))(\beta-\alpha+\beta /(4 \beta+1))} .
\end{aligned}
$$

Adding $j^{-2} N_{j}^{\beta} f\left(s, N_{j}, a_{j}\right)$ we get a series (1) for which $\mu(0) \leq \beta$ (actually, $\mu(0)=\beta)$ and

$$
\mu(\alpha) \geq \frac{4 \beta+1}{6 \beta+2}\left(\beta-\alpha+\frac{\beta}{4 \beta+1}\right) .
$$

The particular form of the second member is not very exciting. What is important is that $\mu(\alpha)>0$ whenever $\alpha<\beta+\beta /(4 \beta+1)$ that is

$$
\omega_{\mu} \geq \beta+\beta /(4 \beta+1) \text {. }
$$

When $\beta$ is small, the second member is $2 \beta(1+o(1))$, therefore

$$
\mu^{\prime}\left(\omega_{\mu}-0\right)>-(1+o(1)) / 2 \text {. }
$$

Let us state the result.

THEOREM 3.3. There are Dirichlet series (1) for which the Lindeloff function $\mu(\sigma)$ has slopes $\left|\mu^{\prime}(\sigma)\right|$ as near $\frac{1}{2}$ as we want.

One can get a slight improvement in (110) by using the lemma in a different manner, taking $l=d=d(N)$ (small when $N$ is large). However there is no reason to believe that it provides the best estimate.

A new idea would be necessary for the third step, namely, the analogue of the second step with $\beta>1$.

\section{References}

[1] H. Bohr, Bidrag til de Dirichlet 'ske Raekkers Theori, (Dissertation, Copenhagen 1910) (IA3 and in English translation III SI in Collected Mathematical Works of Harald Bohr)

[2] H. Bohr, 'On the convergence problem for Dirichlet series', Dan. Mat. Fys. Medd. 256 (1946), 1-18. (IA17 in Collected Mathematical Works of Harald Bohr) 
[3] H. Bohr, 'Zur Theorie der Dirichletschen Reichen', Math. Z. 52 (1950), 709-722. (IA18 in Collected Mathematical Works of Harald Bohr)

[4] H. Bohr, 'On the summability function and the order function of Dirichlet series', Dan. Mat. Fys. Medd. 274 (1952), 1-39. (IA22 in Collected Mathematical Works of Harald Bohr)

[5] G. H. Hardy and Marcel Riesz, The general theory of Dirichlet's series, (Cambridge Tracts in Math. and Math. Physics 18 (1945).

[6] H. Helson, 'Convergent Dirichlet series', Ark. Mat. 4 (1962), 501-510.

[7] H. Helson, 'Convergence of Dirichlet series', L'analyse harmonique dans le domaine complexe, (Table Ronde, C.N.R.S., Montpellier 1972, Lecture Notes in Math., vol. 336, Springer-Verlag 1973, pp. 153-160).

[8] J. P. Kahane, 'Sur les series de Dirichlet $\sum_{1}^{\infty} \pm n^{-s}$, C.R. Acad. Sci. Paris 276 (1973), 739-742.

[9] J. P. Kahane, 'Sur les polynomes à coefficients unimodulaires', Bull. London Math. Soc. 12 (1980), 321-342.

[10] H. L. Montgomery and R. C. Vaughan, 'Hilbert's inequality', J. London Math. Soc. 8 (1974), 73-82.

[11] H. Queffelec, 'Propriétés presque sûres et quasi-sûres des séries de Dirichlet et des produits d'Euler', Canad. J. Math. 32 (1980), 531-558.

\section{Département de Mathématique \\ Université de Paris-Sud \\ 91405 Orsay}

France 